\title{
Las regiones transfronterizas en América Latina
}

\author{
Haroldo Dilla Alfonso
}

Universidad Arturo Prat, Iquique, Chile. Email: hdillaalfonso@gmail.com

\section{Ingrid Breton Winkler}

Universidad Arturo Prat, Iquique, Chile.

Email: ibretonwinkler@outlook.com

Resumen: El surgimiento de regiones transfronterizas es uno de los signos más importantes de los procesos de articulación fronteriza en el capitalismo neoliberal. Ellas actúan como espacios de valorización del capitaly de disciplinarización de los otros en función de la acumulación económica y el poder político. El artículo discute estos presupuestos desde la perspectiva latinoamericana para lo que propone cuatro tipos de regiones transfronterizas a partirde las escalas de relacionamiento económico de ellas. Culmina con algunas consideraciones hipotéticas para un debate más sostenido sobre el tema.

Palabras clave: Fronteras, ciudades, regiones, regiones transfronterizas

\section{Cross-border regions in Latin America}

\begin{abstract}
The emergence of cross-border regions is one of the most important signs of border articulation processes in neoliberal capitalism. Such regions act as spaces for the valorization of capital and the disciplining of others in terms of economic accumulation and political power. This article discusses these assumptions from the Latin American perspective, proposing four types of cross-border regions based on their scales of economic relations. It culminates with some hypothetical considerations for a more sustained debate on the subject.
\end{abstract}

Keywords: Borders, cities, regions, cross border regions

\section{As regiões transfronteiriças da América Latina}

Resumo:O surgimento de regiões transfronteiriças é um dos sinais mais importantes dos processos de articulação fronteiriça no capitalismo neoliberal. Eles atuam como espaços para a valorização do capital e o disciplinamento dos demais em termos de acumulação econômica e poder político. O artigo discute essas premissas da perspectiva latino-americana para o que propõe quatro tipos de regiões transfronteiriças a partir das escalas de relacionamento econômico das mesmas. Termina com algumas considerações hipotéticas para um debate mais sustentado 
sobre o assunto.

Palabras-chave: Fronteiras, cidades, regiões, regiões transfronteiriças

"Los lugares entre lugares

Son como pequeños países

ellos mismos, con sus propias fiestas”

(Alberto Ríos: “Día de los refugios”)

El tema a que nos convoca Polis -la construcción fronteriza en los procesos de territorialización latinoamericana- es un asunto relevante del que las ciencias sociales regionales han dado cuenta de manera fructífera. En particular, la contraposición entre (re)fronterización ${ }^{1}$ y des-fronterización ha sido un dilema heurístico tan relevante como socorrido en los estudios especializados. Y aún cuando ello ha sido hecho desde perspectivas diferentes -históricas, sociológicas, etnográficas, etc.-lo que reúne a la mayor parte de ellos ha sido la asunción de esta relación desde una percepción binaria en que regularmente la desfronterización aparece asociada al contacto y su némesis, la refronterización, a la separación.

Una primera forma de tratar el asunto ha partido del enfoque histórico.Aquí, el dilema ha sido usado para definir dos situaciones diacrónicas. Se trataría del contraste entre las fronteras duras westfalianas y las fronteras dinámicas que impone la globalización neoliberal. En el primer caso se trata de los límites rígidos que maduraron en los estados nacionales y resultaron óptimos respecto a las necesidades del capitalismo fordista del siglo XX. Fueron las típicas fronteras percibidasy usadas como trincheras de contención que garantizaban la exclusión de aquellas variables exógenas -económicas, políticas, sanitarias, ideológicas, identitarias, etc.consideradas lesivas a los sistemas nacionales. Y fueron, finalmente, los temas de investigación de la “fronterología” geopolítica que tuvo sofisticados exponentes en los trabajos de Glassner y de Blijt (1980), Prescott (1987) Nweihed (1990) y Foucher (1991), entre otros.En el segundo caso, las fronteras pasan a ser líneas que cruzan los factores del intercambio económico - mercancías, capitales, personas, informaciones- al calor de los avances de la mundialización capitalista, fronteras evanescentes, según Ohmae (1991).El resultado ha sido un anaquel repleto de estudios y posicionamientos teóricosregularmente inspirados en la experiencia europea y, en sus primeros momentos, en el acuerdo de libre comercio norteamericano.

Según las predicciones de Ohmae(1991) perdieron credibilidad, se abrió camino una percepción más compleja de las fronteras como dispositivos contradictorios y multidimensionales. El dilema des/re/fronterización 
comenzó a ser explicado como un proceso sincrónico, que contenía en sí mismo la relación contacto-separación. Ello ha generado un área de investigación -una nueva modalidad de "fronterología”- que gana espacio en las ciencias sociales y ha permitido percibir este asunto como una situación múltiple y dinámica que rebasa el nicho de las fronteras internacionales. Un claro indicio de lo que Bauman (2002) argumentara al comenzar el siglo:"el espacio global ha adquirido el carácter de un espacio de fronteras”(p. 116)

Sin embargo, el valor epistemológico de estos avances ha sido limitado -en lo que a las fronteras internacionales concierne- por la prevalencia no sólo de un enfoque binario antes comentado, sino también de un cierto toque ontológico que tiende a priorizar el carácter autorreferente (Luhman, 1997) ${ }^{2}$ de los sistemas fronterizos. Y en consecuencia, las fronteras son aprehendidas de manera fragmentada como sujetos -ciudades, regiones- que se expresan a través de políticas públicas, estrategias económicas o pactos con homólogos isomórficos ubicados a los otros lados de la línea.

Una presentación argumental típica en este sentido ha sido proveída por Herzog y Sohn (2014) en un artículo que tuvo una notable y merecida difusión en los cenáculos académicos y sobre el cual queremos detenernos a modo de ejemplo. La desfronterización -según los autores- fue el signo predominante durante los 90s, cuando, sobre las ruinas del Muro de Berlín, se produjeron cambios tecnológicos y políticos que potenciaron "el contacto y el intercambio" (las comunicaciones, el transporte y la voluntad integracionista) a niveles inéditos. Y en relación con ello, el uso de las fronteras como recursos, argumento este último que había sido desarrollado por Sohn (2014) anteriormente. Pero esta tendencia fue contrarrestada desde el presente siglo por otra de refronterización marcada por el discurso de la seguridad. La presente situación que denominan fronterización, por tanto, se define desde el cruce contradictorio de tendencias de signos opuestos pero "intrínsecamente complementarias", lo que los autores organizan en una serie de "categorías analíticas". El estudio busca una base teórico-metodológica apta para discutir las oportunidades y perjuicios que ofrece la fronterización a dos "metrópolis transfronterizas": el complejo Tijuana/San Diego y la región metropolitana de Ginebra, en lo que logran conseguir interesantes análisis de casos.

El punto débil de toda esta argumentación es el relegamiento a un plano subsidiario de lo que en realidad es la esencia del problema: la manera como las fronteras internacionales intermedian en (y por consiguiente contribuyen a la articulación de)los procesos de acumulación económica, de reproducción del poder político y de despliegue de prácticas sociales vitales para el funcionamiento sistémico. Y, en consecuencia, no consiguen explicar lo que proclamaron al inicio de su escrito como un vector conceptual: la relación conflictiva entre losespacios de los flujos y losespacios de los lugares que Castells (1989) y Borja y Castells (1997) se encargaron de instalar en los estudios urbanos con probada efectividad teórica ${ }^{3}$. El resultado de ello es que Herzog y Sohn (2014) terminan discutiendo el binomio 
contacto/separación como una diada esencial, cuando en realidad es una manifestación fenoménica.

No se trata, aclaramos, de que la contraposición entre contacto y separación, sea un dilema supernumerario en cualquier región transfronteriza. Basta observar los dramas humanos que ocurren en fronteras atestadas de aspirantes al paso o los intensos programas de control de las "nuevas amenazas" para entender que ello sigue siendo un dato crucial de las fronteras contemporáneas. La tentación a resucitar muros de contención -lo que Rosiere y Jones (2012) calificaban como teichopolítica- es una realidad lamentable común en el mundo contemporáneo. Solo que todo ello -no importa ahora cuan mediáticas y escandalosas resulten- son una expresión fenoménicaen un nuevo contexto en que las fronteras se articulan como mecanismos de disciplinarización/normalización de la diferencia,de una modalidad de control de los individuos y de los procesos en el tiempo y en el espacio (en términos de Foucault, 2003) y filtros para garantizar los procesos de intercambio desigual.

En última instancia, por el paso fronterizo de San Ysidro que separa San Diego de Tijuana -marcado por un muro doble con casamatas y patrullas especializadas- se producen cada año unos 40 millones de cruces de personas en ambas direcciones, y aproximadamente la mitad de los habitantes de Tijuana están habilitados para realizar estos cruces diariosy 6,5 millones de cruces anuales animan al paso de Chacalluta/Santa Rosa que conecta a Tacna y Arica y que resulta la única vía disponible en unos 170 kilómetros de un límite severamente vigilado yatestado de minas antipersonales.

La caracterizaciónde la frontera como filtro -que Liberona desarrolló agudamente para la experiencia del Norte Grande chileno- fue originalmente argumentada por Kearney (2008). Aunque su foco era las migraciones humanas, no es difícil extrapolar a un contexto más amplio que involucra a los otros temas clásicos de las fronteras: seguridad, comercio, inversiones, cultura, medio ambiente, etc. Toda frontera, afirmaba Kearney, tiene dos misiones clasificatorias esenciales: la que cataloga según la dimensión étnico-cultural, y la que se centra en la condición clasista. Ambas clasificaciones se refuerzan mutuamente, en un juego dialéctico que recuerda poderosamentela manera como Nancy Fraser (2003) discutió con Axel Honneth la relación existente entre la redistribución y el reconocimiento, y que el autor asume como una reinterpretación antropológica de la teoría del valor de Marx. Por consiguiente, afirma Kearney (2008),

Las políticas y las prácticas fronterizas de un estado naciónpueden considerarse un recurso para obtener un flujo neto de valor económico a través de su frontera, y hacia su territorio, por medio de la clasificación en los dos sentidos mencionados (Kearney, 2008. p.81).

Colocar este dilema a debate ha sido el mérito de la obra de Mezzadra y Neilson (2013) cuando se referían a la creciente disimilitud entre “...la expansión de las fronteras del capital y la proliferación de límites legales, 
políticos y sociales que nos informan de las fronteras como método.” (p. 80). Afirmamos, escribían Mezzadra y Neilson (2013)

"que las fronteras son igualmente mecanismos de inclusión que seleccionan y filtran personas y diferentes formas de circulación de maneras no menos violentas que aquellas desplegadas en medidas excluyentes”. Y lo hacen a través de “...la multiplicación de conexiones y desconexiones territoriales, económicas, sociales y culturales” que define la “...capacidad de jerarquización y estratificación de los bordes” (Ibíd., p. 7).

Sería erróneo creer que el lugar de ejercicio de este proceso de disciplinarización es el puesto fronterizo, un "emplazamiento funcional" diría Foucault saturado de funcionarios adustos. Este es solo un lugar de condensación de controles primarios que son ejercidos, en primera instancia, en una articulación espacial multidimensional y multiescalar que aquí denominaremos -siguiendo a Jessop (2001)- regiones transfronterizas (RTF).Estas regiones serán nuestro objeto de análisis en este artículo. En primer lugar discutiremos algunas aristas conceptuales en torno al tema, para luego proceder a proponer un análisis de diferentes situaciones regionales transfronterizas en relación con la reproducción y la acumulación económica en América Latina.Y finalmente, adelantaremos algunas reflexiones preliminares acerca de los retos heurísticos que estas regiones comportan.

El artículo se organiza a partir de una metodología deductiva desde informaciones propias de los autores en trabajos sobre el tema en varias situaciones fronterizas, así como la lectura y discusión de una notable producción académica latinoamericana que habla de la pujante relevancia del tema más allá de los nichos geopolíticos. No obstante, como los lectores notarán, las ideas que aquí exponemos tienen un tono polémico y, de hecho, son presentadas de una manera ensayística. Ello persigue un objetivo vital para el trabajo académico e intelectual: incentivar el debate como herramienta epistemológica, calistenia inseparable de aquello que Morin (2000) llamaba "el derecho a la reflexión en contraposición a la confrontación ciega de los hechos o la verificación testaruda de hipótesis fútiles”(p. 59)

\section{Las regiones transfronterizas como territorialidades distintivas}

Las regiones -entendidas aquí como ensamblajes territoriales a escalas geográficas diferentes de las escalas nacionales- constituyen sistemas espacio/temporales que Harvey (1996) definía como "artefactos materiales ecológicos y redes intrincadas de relaciones sociales” (p. 265).En cuanto sistemas complejos, se articulan desde la interacción de factores materiales, sociales y mentales -políticas estatales de regulación y habilitación, culturas territoriales, tradiciones comunitarias- que invitan a pensar- 
las desde elinstrumento heurístico de la sobredeterminación(Althusser, 1974). Existen, por tanto, regiones articuladas desde las tradiciones y las existencias comunitarias ancestrales, u otras basadas -recordando a Tilly (1990)- en los ámbitos de la coerción. Pero lo que distingue a las regiones contemporáneas son sus remisiones -en última instancia- a la existencia de una economía política territorial: la "coherencia estructurada de la producción y el consumo”, es decir “...el espacio en el que el capital puede circular sin que el coste y el tiempo de movimiento excedan los límites del beneficio impuestos por el tiempo de rotación socialmente necesario” (Harvey, 2014, pp. 348-349).

En la misma medida en que el régimen de acumulación portfordista entraña una alta movilidad del capital en relación con otros factores territorializados “fijos”(los estados y las comunidades), su dinámica reta a la territorialidad nacionalista y pone en jaque a la doxa westfaliana de pueblos cuyos límites sobre los territorios coinciden con los límites del estado. Ello genera lo que aquí denominaremos Regiones Transfronterizas (RTF), y que definiríamos como sistemas espacio/temporales multidimensionales ymultiescalares que abarcan territorios contiguos bajo jurisdicciones nacionales diferentes.

Una mirada seminal al respecto fue un artículo, escrito por Bob Jessop (2001), que llevó como título "La economía política de la escala”. Curiosamente la mayor parte del artículo está dedicada a un extenuante ejercicio taxonómico de los procesos de regionalización -cuatro tipos de articulación escalar, una docena de formas de re-escalamiento y nueve formas de emergencia- que hoy nadie podría repetir sin pedir disculpas, entre otras razones por su acendrado europeísmo. El punto fuerte de la argumentación de Jessop -y que convirtió su artículo en fuente obligada- fue su énfasis en que los procesos de reescalamiento que conducían a las RTF estaban condicionados por los cambios en los regímenes de acumulación y la consiguiente erosión de la escala nacional distintiva de los predios del capitalismo fordista, pero que no podían ser considerados "territorios económicos espontáneos y naturales" sino construcciones sociales derivadas de luchas sociales y discursivas, pujas institucionales y otras acciones en que la escala global es decisiva en muchos sentidos pero atenida a la interacción con otras escalas y sus actores. Y en particular eran "objetos específicos de políticas” (p. 37) en este nuevo ensamblaje marcado por la desnacionalización de los estados, la desestatización de la cosa pública y su gerencia desde vínculos funcionales y responsabilidades compartidas:

Tanto en términos geoeconómicos como geopolíticos, los tipos variados de regiones están marcados por grados de hegemonía y jerarquía diferentes y cambiantes, superponiéndose esferas de influencia, componentes nacionales e influencias transnacionales, interdependencias y paquetes de regiones autocontenidas, embrionarias y fallecientes, esferas marginales y áreas de confrontación. Estas complejidades proveen más oportunidades para el reescalamiento, el salto entre escalas... ellas también reordenan las 
jerarquías espaciales y escalares, produciendo nuevas formas de desarrollo desigual. Esto se refleja no sólo en cambios entre las "economías nacionales”, sino también en el auge y declinación de regiones, en nuevas formas de división "norte-sur" y así sucesivamente (Bob Jessop, 2001,p. 36).

En este sentido, las RTF devienen una forma específica de solución espacio/temporal que subvierte territorios hasta el momento vedados a la acumulación por razones geopolíticas o de economía de escala. La nueva territorialidad ofrece a la acumulación nuevas oportunidades, sea por la oferta de posibilidades de consumo productivo, por la reducción de costos (o por el incremento de las tasas de rentabilidad del capital a partir del uso de los precios diferenciales.

En la misma medida en que las RTF maduran como laboratorios institucionales neoliberales (Brenner y Theodore, 2002)y filtros para garantizar "los procesos de intercambio desigual” (Kearney, 2008, p. 81), ocurren en su interior complejos procesosde administración de la diferencia y el conflicto. No se trata solamente de aquella situación de "territorialidades superpuestas” que Agnew y Oslander (2010) percibían desdela emergencia de actores no estatales que disputaban soberanía al estado. Aquí también ocurre que esas disputas por autoridad y recursos se realizan entre diferentes sistemas políticos y socio/ culturales. Desde esas colisiones conflictivas los trabajadores devienen ilegales, las mercancías se trocan en contrabando y las culturas en folclor y las propios espacios transfronterizos en lo que Balibar (2013) llamó “zonas subordinadas de sacrificio” vitales para la acumulación a diferentes escalas.

Los estudios especializados de fronteras en América Latina han frecuentado las ciudades fronterizas. Sea desde perspectivas históricas, sociológicas o espaciales, existen numerososestudios que han generado una interesante colección de términos -no siempre remitidos a definiciones conceptuales, ni felices en sus propias connotaciones- queincluye denominaciones tales como"metrópolis transfronterizas” (Herzog, 1990); "sistemas metropolitanos binacionales" (Urdaneta, 2002); "ciudades postfronterizas" (Dear y Leclerc, 2003); “ciudades transfronterizas” (Valero, 2004 y 2008); “conurbaciones fronterizas", "región transnacional” y "corredor transnacional” (Peña, 2008); “ciudades gemelas” (Ministerio de Integración Nacional/ Grupo RETIS, 2005 y Machado de Oliveira, 2005) y “complejos urbanos transfronterizos” (Dilla, 2015), entre otras. Todos estos estudios constituyen aportes signficativos, pero limitados, para el tema que nos ocupa, toda vez que las ciudades que se ubican sobre las fronteras -en ocasiones en colindancia con otras al otro lado del borde en aparente relación isomórfica- son regularmente partes de redes más extensas que incluyen polos urbanos dominantes, ciudades auxiliares y áreas no urbanizadas que tributan a la condición transfronteriza.

Desafortunadamente, los estudios regiones (trans)fronterizas en la complejidad antes mencionada, han sido poco usuales en los estudios lati- 
noamericanos y en ocasiones han estado lastrados por la percepción europeístaque define al pacto como punto de inicio de la regionalización (Perkman, 2003), lo que se manifiesta enOddone, Quiroga, Sartori y Williner (2016) y Barajas, Wong y Oddone (2015), entre otros. Vale mencionar, sin embargo, otras aproximaciones emanadas desde estudios empíricos concretos y que han significado avances heurísticos en la dirección que nos interesa, como son los casos de los estudios desarrollados en la frontera brasileña por el grupo RETIS (Ministerio de Integración Nacional/RETIS, 2005) y por Machado (2005); en la frontera colombiana/ amazónica por Zárate, Aponte y Victorino (2017), en la frontera de Venezuela con Colombia y Brasil (Valero, 2009), en la frontera dominico/haitiana (Dilla, 2010)y en el borde Perú/Chile (Dilla y Álvarez, 2018).

En particular -por sus magnitudes e intenciones explícitas de tipologización- resultan interesantes para nuestros fines los estudios desarrollados en Brasil al calor del diagnóstico de franjas fronterizas que el Ministerio de Integración Nacional encargó el Grupo RETISde la Universidad Federal de Río de Janeiro, bajo la coordinación de Lía Machado. En el diagnóstico se realiza una pormenorizada construcción conceptual en torno a las territorialidades fronterizas que se detiene en las regiones descriptas como” espacios dotados de coherencia funcional, política y simbólica” (p. 94). Aunque desafortunadamente -y debido a la naturaleza del propio estudio- el equipo RETIS solo prestó atención referencial a las zonas fronterizas colindantes con las brasileras, por lo que las regiones que definen no son espacios propiamente transfronterizos.

Estas regiones fueron clasificadas en tipos atendiendo tanto a sus situaciones internas como a los grados de interacción transfronteriza, lo cual fue evaluado a partir de diversas variables como los comportamientos de los mercados laborales, los flujos de capitales, la disposición de recursos naturales, la calidad de los servicios de consumo colectivo y la habilitación infraestructural, entre otros factores. Ello implicaba una interesante perspectiva multidimensional que involucraba factores económicos, políticos, ambientales, etc. Finalmente resumieron cinco tipos: regiones marginales (con interacciones muy poco intensas y eventuales); zonas-tapones (caracterizadas por bloqueos espaciales e institucionales que dificultan o impiden el acceso); frentes (áreas de poblamiento y colonización); capilares (se basan en intercambioslocales) y sinápticos (cuando implican relacionamientos mayores que trascienden las escalas locales). Estas modalidades de regiones, cuando se ubican en los tipos más activos, afirman los autores, incluyen la existencia de lo que denominan "ciudades gemelas" cuyas relaciones y simetrías con frecuencia tienen en el ámbito regional sus condicionamientos principales ${ }^{4}$.

Esta clasificación adolecía del problema antes mencionado de su reclusión en una sola parte de la frontera y de una cierta ambigüedad en la definición de los tipos. No es difícil advertir, por ejemplo, que tras la categoría sináptica se esconden diversas modalidades de interacción económica, tanto en cuanto a dimensiones de relacionamientos como en referencia a las 
escalas en que son asumidos y que pueden resultar más relevantes que otros tipos residuales como las llamadas zonas marginales o tapones.

La aproximación de Machado de Oliveira (2005) estaba evidentemente impregnada de los estudios antes mencionados de RETIS-de los que el autor fue parte- pero asumía como eje metodológico la dicotomía entre dos situaciones: la integración formal (institucional) y la funcional (principalmente de intercambios económicos). Esta dicotomía fue plasmada sobre el recurso lógico de un eje de coordenadas que daba lugar a cuatro tipos de situaciones regionales:

1. Situación A de baja integración formal y funcional, lo que denomina una frontera muerta o “marginal” según la tipología de RETIS.

2. Situación B de baja integración formaly alta integración funcional que remite a la ilegalidad, la informalidad y la anomia.

3. Situación C de alta integración en los dos sentidos, que denomina "fronteras vivas" con altos flujos de intercambios regidos por un alto nivel de institucionalización. Las remite parcialmente a las fronteras sinápticas de RETIS (Machado, 2005).

4. Situación D de baja integración funcional y alta formal, lo que define como fronteras burocráticas.

La clasificación de Machado (2005) fue un paso adelante de primer orden en el tema que nos ocupa y sigue siendo, tres lustros después, un punto de referencia teórica sobre las regiones en las fronteras latinoamericanas. Pero sus virtudes no omiten sus puntos débiles. En primer lugar, persistió en anclar su análisis en un lado de la frontera aúncuando hubiera podido adelantar, al menos hipotéticamente, algunas ideas sobre la dimensión transfronteriza. En segundo lugar, Machado asumió un compromiso metodológico con el dilema formal/informal que había alcanzado una distinción de doxa académica en los estudios de fronteras a partir de las experiencias europea y norteamericana y que fue alentada desde los centros académicos del norte mexicano. Y no tomó en cuenta lo que ya había sido advertido en varios estudios especializados: la formalización de los espacios de fronteras en América Latina no haconducido a situaciones virtuosas en función de las realidades transfronterizas ${ }^{5}$.

\section{Las situaciones de regionalización transfronteriza en América Latina}

En América Latina no existe ningún proyecto integracionista que se haya propuesto la superación de los estados nacionales, como es el caso europeo. Los proyectos de integración existentes -con la excepción en alguna medida de la Comunidad Andina de Naciones- ni siquiera han intentado enfoques específicos hacia las fronteras más allá de sus habilitaciones 
como lugares que deben facilitar el tráfico de mercancías. Por lo tanto, no encontramos en el continente procesos significativos y perdurables de institucionalización transfronteriza más allá de acuerdos básicos y pragmáticos para agilizar algunos procesos o acceder a recursos, como son los casos de los Comités de Integración y Desarrollo Fronterizo en el cono sur y de las Zonas de Integración Fronteriza en el área andina.

Pero al mismo tiempo, y a contrapelo deesta carencia,se puede afirmar que las fronteras latinoamericanas han asumidoincrementos sustanciales de flujos de personas, capitales, mercancías, etc., lo que ha suscitado diversas expresiones de regionalización transfronteriza. Estas regiones son multidimensionales, en la medida que en su formación y desarrollo inciden factores diversos (culturales, geopolíticos, ambientales, económicos) entre los cuales, anotábamos antes, los económicos ejercen regularmente una determinación de última instancia. En ningún caso estas territorialidades transfronterizas rebasan lo que Sohn (2014) llamó un modelo geoeconómico de explotación de ganancias diferenciales.

A partir de esta consideración, proponemos una tipologización de las regiones transfronterizas latinoamericanas a partir de las conexiones de estas a una escala económica que rige el ámbito de los intercambios económicos, y por tanto le distinguen.

Teniendo en cuenta que en cualquier RTF existe una superposición de escalas, toda definición al respecto puede resultar imprecisa. Para citar un ejemplo conocido podemos remitirnos a la región transfronteriza que se articula en torno a la ciudad de Cúcuta en Colombia y la red urbana que centraliza San Cristóbal en Venezuela. Esta región se ha caracterizado históricamente por una notable compartición cultural y étnica, lo que aún se expresa en las percepciones mutuas y en intercambios menores en las zonas rurales. Al mismo tiempo, sus espacios urbanos han sido escenarios de fuertes flujos de comercio local cuyas direcciones han variado según las situaciones económicas de cada lado y los vaivenes de las tasas de cambio monetarias.Finalmente, hasta la crisis venezolana, este corredor constituía uno de los pasos comerciales terrestres más activos del continente, con unos 3,6 mil millones de dólares en 2006, lo que impacta de diversas maneras en el espacio regional. Al referirse a esta multiplicidad escalar y dimensional, Valero (2008)afirma que

Constituyen el origen/destino de los intereses intersocietales, derivados de exigencias y necesidades -algunas locales, otras regionales y nacionales, e incluso del impacto global del mundo contemporáneo- manifiestos mediante... redes y flujos fortalecedores de la dinámicay, en consecuencia, de la interdependencia binacional (Valero, 2008, p. 74).

Pero estascomplejidades y yuxtaposiciones escalares no omiten que existandeterminaciones fundamentales que organizan a la totalidad sistémica y que en última instancia responden a la pregunta de cómo se manifiesta la 
coherencia estructurada de la economía política regional. Y desde aquí, también como se ordenan las diferentes dimensiones de relacionamientos transfronterizos a partir de la manera como la región realiza su función de filtro y de disciplinamiento de factores y procesos tales como la movilidad humana, los tráficos de mercancías, las relaciones estatales y las simbiosis culturales.

Desde estas consideraciones es posible pensar en cuatro tipos de situaciones fronterizas que, repetimos, priorizan como variables de clasificación el lugar de ellas en los procesos de acumulación.

- Un primer tipo corresponde a lo que aquí denominaremos como regiones consuetudinarias, entendidas como aquellos sistemas espacio/temporales sustentados en comparticiones de valores ancestrales y en relaciones familiares y étnicas. Incluyen relaciones mercantiles, pero de montos menores y en ocasiones sin mediaciones monetarias. En la misma medida en que son regiones poco pobladas y periféricas en términos geopolíticos, estas regiones tienen niveles muy bajos de institucionalización formal, aunque la presencia represiva de los estados puede ser alta cuando ocurren conflictos locales, en particular cuando las identidades subalternas retan las primacías nacionalistas.

Es el caso de situaciones de identidades étnicas violentadas por las imposiciones nacionalistas, como ocurre en la territorialidad mapuche que rebasa el límite argentino/chileno, o en los espacios andinos aymarás que incluye partes de las actuales Bolivia, Chile y Perú. Otro caso de esta naturaleza puede estar explicado en el exhaustivo estudio de Zárate, Aponte y Victorino (2017) sobre la frontera amazónica de Colombia con Perú y Brasil, donde los autores encontraron flujos mercantiles menores, una parte muy significativa de los cualeseran trueques no monetarios de mercancías extraídas del ambiente inmediato, por lo que no aparecen en los conteos estadísticos. En consecuencia, aun cuando muy relevantes para los contextos locales, estas cantidades "son muy poco significativas en relación con las magnitudes y los aparatos productivos de cada país o con los de la región suramericana en su conjunto” (Victorino, 2017, p. 76). Un elemento clave de cohesión regional lo constituye "la intensidad de las relaciones sociales y de parentesco" (ibíd., p. 69) a partir de las identidades étnicas (ticunas, muruis, etc).

- Una segunda situación correspondería a aquellas regiones en que los intercambios económicos se resuelven localmente, y que aquí denominamos como regionesautocontenidas.No son necesariamente regiones fallecientes -en el sentido planteado por Jessop (2001)y de hecho, algunas de ellas pueden mostrar flujos muy dinámicos que rebasan con mucho la parquedad mercantil de las regiones consuetudinarias. Tampoco son autárquicas, pues todas ellas tienen entradas y salidas en relación con otras escalas, pero lo hacen de 
manera indirecta y resultan poco relevantes para esas otras escalas. En consecuencia, se caracterizan por cadenas de valores cortas y de valores agregados exiguos. Los controles fronterizos varían de intensidad de unos a otros casos, según la magnitud de los intercambios, pero siempre implican un nivel alto de permisividad, en la misma medida en que en estas regiones los circuitos económicos adquieren la forma de flujos de movilidad humana, y en consecuencia de acuerdo con Certeau (2008) las prácticas sociales devienen “prácticas organizadoras” de los espacios urbanos y sus ámbitos de relacionamientos transfronterizos. Huelga anotar que también aquí los vínculos étnicos y de parentesco tienen un rol muy importante como garantías de lealtad en negocios que transcurren sin contratos formales.

Esta situación regional es típica decomplejos urbanos transfronterizos de dimensiones pequeñas y medias con espacios colindantes como son Chuy oChui en la frontera brasilera/uruguaya (Domínguez, 2010); Dionisio Cerqueira,Barracao y Bernardo Irigoyen en Argentina/Brasil (Ferrari, 2013); Leticia y Tabatinga en la frontera amazónica de Colombia y Brasil (Nogueira, 2008); y el trío Monte Caseros/Barra de Quarai/Bella Unión (Oddone, 2015).

Es también el caso de las ciudades de Hidalgo y Tecún Umán, en el borde Guatemala/México, estudiado por Ruíz y Martínez (2014).Los autores ofrecen una vívida descripción de los procesos de intercambios entre dos ciudades medianas(en total no superan los $70 \mathrm{mil}$ habitantes) a partir de las compras que los comerciantes guatemaltecos hacen en Ciudad Hidalgo para abastecer los comercios de Tecún Umán y alimentar flujos menores dirigidos a otras localidades. El tráfico -realizado sobre el río Suchiate- involucra diariamente a cientos de personas entre comerciantes, cargadores, remeros de balsas rústicas, cambias, etc., lo que implica una sucesiva negociación y puesta de acuerdo sobre precios y servicios. En la misma medida en que el tráfico afecta ambas partes, los actores involucrados -pequeños empresarios, autoridades municipales, trabajadores- han conseguido acuerdospara alternar la prestación de servicios. En consecuencia, apuntan los autores, “...la conurbación Ciudad Hidalgo-Tecún Umán constituye un espacio integrado de intercambios económicos y sociales, basados fundamentalmenteen el eje conformado por el comercio transfronterizo informal” (Ruíz y Martínez, 2014, p 152).

Pero también es posible encontrar una región autocontenida en espacios más poblados y dinámicos como es el complejo urbano transfronterizo Tacna-Arica en la frontera chileno-peruana (en total algo más de medio millón de habitantes) cuya relación económica induce unos 6,5 millones de cruces de personas cada año y que ha sido estudiada por Dilla y Álvarez (2018). Aunque esta región se alimenta de importaciones proveídas por zonas francas comerciales, 
la relación entre ambas ciudades implica numerosos circuitos -mano de obra peruana en Arica, usode Tacna como playa de ocio y bazar por los ariqueños, uso del puerto de Arica por comerciantes tacneños, etc.- las incidencias de esta actividad en los espacios nacionales respectivos es muy limitada, sin que tampoco podamos hablar de una inserción global significativa.

- Una tercera situación correspondería a las regiones articuladas en torno a corredores internacionales y que se definen fundamentalmente desde él. Toda región transfronteriza implica algún tipo de corredor que canaliza los flujos de mercancías, regularmente sobre alguna infraestructura vial habilitada para este fin. Pero debe hacerse notar que los corredores comerciales binacionales no conforman necesariamente regiones, o pueden hacerlo de manera muy deficitaria. Para estos corredores los emplazamientos fronterizos son no-lugares (Augé, 1992).

En ocasiones estos corredores cruzan las fronteras por páramos despoblados, como ocurre en el paso de Los Libertadores que constituye la principal conexión fronteriza Chile/Argentina. Según Aduanas de Chile (2017) por este paso se produjeron, en ambas direcciones, 600 mil cruces de vehículos, 2,9 millones de cruces de personas y 4,5 millones de toneladas de carga. Una parte de esta carga de mercancías era comercio binacional, pero otracorrespondía a mercancías argentinas en tránsito hacia los puertos chilenos de San Antonio y Valparaiso. Obviamente este tráfico -intenso y distante- afecta a una extensa zona de ambos países, pero no logra constituir nada parecido a una región transfronteriza debido a que toda la zona aledaña al límite corresponde a una agreste región cordillerana solo animada por los establecimientos de control fronterizosy algunos moteles estacionales.

Otro caso es el de Jimaní en República Dominicana, una ciudad particularmente pobre en la frontera de este país con Haití. Se le calcula una población solo ligeramente superior a los 10 mil habitantes, el 50\% de los cuales son pobres. Sinembargo, por ella transita el $60 \%$ del comercio binacional, una suma que debe ascender a unos 500 millones de dólares anuales. La ciudad solo resulta un lugar de paso para las caravanas de camiones que dejan detrás todo tipo de contaminación, muy pocos empleos formales y algunos espacios para la concurrencia de comerciantes que nunca han podido madurar como ferias comerciales transfronterizas. Jimaní incuba el contraste de ser el puerto fronterizo más activo comercialmente, y al mismo tiempo la ciudad cabecera, más pobre de toda la franja fronteriza dominicana, donde la pobreza es una cualidad usual(Dilla, 2010).

Sin embargo, también puede ocurrir que por su magnitud e itinerarios, un corredor involucre de manera significativa la provisión local de servicios y bienes. Y a partir de ahí, induzca otras actividades de intercambios locales. Este es el caso de la región articulada en torno a Corumbá en Brasil y Puerto Suárez en Bolivia estudiado por Silva 
(2013). Por este corredor transita el 95\% del comercio binacional, además de otras mercancías que buscan accesos oceánicos. Excepto algunos productos mineros localizados en Corumbá, los flujos brasileros son alimentados desde grandes ciudades como Sao Paulo, Porto Alegre y Belo Horizonte. Pero la magnitud del tráfico, apunta Silva “...demanda estructuras para el almacenamiento, transporte o despacho de mercancías”(2013, p. 157), lo que genera inversiones, empleos e ingresos fiscales vitales para la sociedad local. Al mismo tiempo que promueve otros contactos económicos que se materializan en las pequeñas ferias libres estudiadas por Anderson, Costa y Benedetti (2017). Son regiones atravesadas por cadenas comerciales largas, con orígenes y destinos distintos a la región en sí, pero que experimentan, al contacto con ella, agregados sustanciales de valores.

Este tipo de región -que denominamos regiones/corredores-subordina la escala local y se organiza desde estrategias de acumulación de grupos capitalistas más concentrados. Dada la magnitud del comercio formal, estas RTF son sometidas a fuertes controles burocráticos y policiales, y son espacios expuestos a conflictos intensos, sea por demandas gremiales, por contradicciones que se generan con los flujos informales o por la proliferación de tráficos criminales.

- Finalmente, un cuarto tipo de regionalización tiene lugar cuando los espacios económicos que se generan están determinados por relacionamientos productivos y de servicios que se definen por sus fuertes tributaciones a la economía global. No es difícil advertir características tales como las altas desigualdades entre las sociedades nacionales -lo que incrementa las ganancias diferenciales- así como altas densidades demográficas, densidades viales y provisión adecuada de servicios. Dada la naturaleza compleja de estos espacios económicos, conviven con niveles muy altos de conflictividad. Son RTF muy institucionalizadas, pero al mismo tiempo muy violentadas por actividades informales e ilegales.

El caso prototípico de esta situación es la extensa frontera México/ Estados Unidos, en que la franja mexicana funciona como un lugar de altísima rentabilidad para las inversiones productivas y de servicios, lo cual ha sido estudiado desde la perspectiva económica de forma exhaustiva por Mendoza (2007).

Otra situación particularmente compleja, y menos conocida, se encuentra en la región trasfronteriza ubicada en el departamento de Alto Paraná en Paraguay y los estados de Matto Grosso do Sur y Paraná en Brasil. Aquí se ha conformado una activa región económica en que el capital brasilero aprovechalas ventajas comparativas paraguayas -desregulación, bajos costos, permisividad- en función de sus propios vínculos con la economía global. Aunque la imagen más mediática de este fenómeno es la gran plaza comercial de Ciudad 
del Este y sus múltiples manifestaciones de contrabando, en realidad incluye procesos más complejos como el cultivo y procesamiento incipiente de la soya y el establecimiento de zonas francas industriales (maquilas), todo lo cual ha sido catalogado por Masi y Falabella (2005) como un desarrollo disgregado que a nivel local "se expresa en partes que no logran armonizarse virtuosamente a pesar de sus enormes logros y potencialidades” (p. 346).

En una dimensión más discreta, pero similar cualitativamente, se encuentra la región que se forma en la porción norte de la frontera haitiano/dominicana, dominada por las segundas ciudades de cada país (Cabo Haitiano y Santiago de los Caballeros) y resumidas en el complejo urbano transfronterizo Dajabón/Ouanaminthe. Nuevamente aquí la visión más mediática es un mercado transfronterizo que dos veces a la semana reúne a varios miles de personas de ambos países, y que tiene un impacto nacional. Resulta, por ejemplo, el principal corredor de productos agrícolas dominicanos a Haití, que los haitianos compensan vendiendo reexportaciones de tejidos usados, perfumes y bebidas alcohólicas. Pero lo que la hace peculiar es que ha servido de locación para el establecimiento de una zona de maquilas dirigidas al mercado mundial que aprovecha las ventajas haitianas (desregulación, baja presión fiscal, fuerza de trabajo muy barata, acceso privilegiado al mercado norteamericano) al mismo tiempo que aprovecha los servicios y el personal técnico más calificado de República Dominicana Dilla (2016).

\section{A modo de conclusiones: ¿Qué nos preguntan las regiones transfronterizas?}

Las territorialidades que hemos llamado aquí regiones transfronterizas han constituido escenarios por excelencia de valorización del capital a diferentes escalas. En particular sus ciudades, siguiendo aquí la reflexión de Brenner y Theodore (2002) resultan "objetivos geográficos y laboratorios institucionales para una variedad de políticas neoliberales”(p. 386), y en consecuencia espacios altamente conflictivos de optimización de la rentabilidad del capital y disciplinarización de los otros. Por estas razones, la noción de frontera ha devenido un recurso heurístico para explicar muchas de las contingencias sociales contemporáneas. Justo lo que Sassen (2010) resumía en un concepto "las zonas fronterizas analíticas", que dan cuenta de la eclosión de nuevas dinámicas espacio/temporales en contextos de significados poco estabilizados. Y en consecuencia, en que la concepción tradicional de territorialidad y de sus jerarquías escalares es retada “...por las prácticas materiales, organizativas y discursivas que entran en juego cuando está en marcha el desplazamiento” (p. 482).

En este artículo se ha intentado discutir algunos de estos retos desde esta perspectiva y la propuesta de una tipología de RTFen América 
Latina en relación con lasescalas de estos procesos. A partir de aquí proponemos cuatro tipos que van desde las regiones consuetudinarias, severamente parroquiales y sin significados económicos más allá de la vida cotidiana de sus moradores, hasta aquellas que se definen desde fuertes vinculaciones con la economía global.

Si exceptuamos al primer tipo mencionado, en él que el intercambio económico es accesorio, lo común de todas estas regiones en nuestro continente es que no rebasan el nivel primario del modelo geoeconómico explicado por Sohn (2014, p. 597) y orientadas a la captura de valores desde los beneficios diferenciales, y que, dado que se realizan casi estrictamente en el mercado, no solo se apoyan en la disparidad de desarrollo, sino que la incrementa.

Se trata, no obstante, de una propuesta provisional cuya finalidad es propiciar un debate que pudiera servir para categorizaciones más complejas que den cuenta de la naturaleza multidimensional de estas territorialidades, como diría Dahrendorf (1979) invernaderos por excelencia de un pluralismo de oposiciones y conflictos.

A modo de inventario polémico, se diría que existentres cuestiones sobre las que las RTF nos interrogan.Una primera cuestión se refiere a la espacialidad de las RTF. Casi todos los estados latinoamericanos declaran zonas fronterizas en las que algunas actividades -por ejemplo propiedades de extranjeros- pueden estar restringidas. Estas zonas se definen regularmente por longitudes desde los bordes, por lo que resultan inapropiadas para precisar la espacialidad de una RTF en los términos en que la hemos definido. Por razones obvias, la dimensión cultural/identitaria de una RTF (allí donde la densidad simbiótica puede producir atisbos de lo que Tarrius (2000) llamaba una identidad mestiza) no coincidirá con la definición política antes mencionada, ni con el ámbito de las transacciones económicas. Esto nos obliga a una definición más pormenorizada de aquellas variables decisivas para la definición de la espacialidad transfronteriza, tomando en cuenta la variedad de dimensiones que las conforman, así como las funciones de los componentes. Probablemente esto nos conduciría a unaespacialidad escalonada más sofisticada -por ejemplo zonas de contigüidad transfronteriza y zonas de convivencia más relajada- que la mirada que hemos ofrecido aquí.

Segundo,se refiere a la cuestión del desarrollo. Difícilmente podemosencontrar en los casos estudiados, experiencias de desarrollo económico local inclusivo. Las regiones transfronterizas son pródigas en sus ofertas de formas de sobrevivencias, pero muy parcas en el ofrecimiento de oportunidades sostenidas de movilidad social. Y ello ocurre no solo en aquellos casos en que las RTF permanecen desconectadas de las dinámicas globales y nacionales - los casos de regiones consuetudinarias y autocontenidas- sino también en aquellas otras severamente expuestas a estas dinámicas y donde se produce lo que Masi y Falabella (2004) reiteran como undesarrollo disgregado. Aún cuando reconociéramos que existe 
unadeterminación sistémica en este resultado -las regiones transfronterizas son zonas de sacrificio de la reproducción neoliberal- es razonable creer que puedan existir espacios para acciones que muevan a estas sociedades en otra dirección.

Tercero, radica en la gobernanza. Como antes anotábamos, no hay en América Latina ningún proyecto integracionista que se proponga una alteración postwestfaliana al actual régimen de fronteras. El proyecto que más ha avanzado en esta dirección es la Comunidad Andina de Naciones, pero a su interior siempre ha existido lo que Hurtado y Aponte (2017) criticaban como una “...cierta descoordinación entre la evolución legislativa y su efectiva implementación local” (p. 71), que conduce a "una institucionalidad... precaria, ineficiente y limitada, así como excluyente en términos de gestión, diseño e implementación” (p. 84). En consecuencia, las RTF demandarían una institucionalidad que de cuenta de la complejidad especial de estas “territorialidades superpuestas” (Agnew y Oslander, 2010), lo que incluye inevitablemente el ensayo de formas de ciudadanías regionales para facilitar la movilidad humana en condiciones de equidad de derechos, lo que Balibar (2013) llamaba un "hecho epistemológico fundamental"' .

En resumen, un tema complejo de sociedades conflictivas, pero donde también se ensayan formas de convivencia que pudieran enseñar mucho en momentos en que el rechazo a los otros parece haberse convertido en signo de buen vivir. Lo decía Alberto Ríos (2017), un poeta de la siempre convulsa frontera de Estados Unidos con México: "El borde es una ecuación en busca de un signo igual”.

\section{Agradecimientos}

Este artículo fue realizado con el auspicio de la Vicerrectoría de Investigaciones, Innovación y Postgrados de la Universidad Arturo Prat. 


\section{Notas}

${ }^{1}$ A lo largo de este artículo usaré el término "refronterización” para aludir a los procesos contemporáneos de fortalecimiento de los controles excluyentes de los otros en escalas internacionales, en lugar de "fronterización". Con ello sigo a varios autores que realizan una contraposición de procesos que explicaré más adelante.

${ }^{2}$ Es decir, como la capacidad de un sistema para establecer relaciones consigo mismos y diferenciarlasde las relaciones con su entorno.

${ }^{3}$ Sólo quemientras los autores primigenios definían estos conceptos desde una visión sistémica del capitalismo contemporáneo -los espacios de los flujos eran totalizadores, dados por las dinámicas de la globalización/mundialización, mientras que los lugares resultaban fragmentados por los primeros ymarcados por la vida cotidiana y las acciones de las comunidades- Herzog y Sohn -en un ejercicio de transmutación que Sartori (1999) hubiera denominado como un "estiramiento conceptual”- reducían ambos a situaciones fronterizas y en particular remitían los “espacios de los lugares” a la proximidad de los bordes mismos. En otras palabras, convirtieron un instrumento heurístico en metáfora.

${ }^{4}$ El diagnóstico socioeconómico mencionado fue complementado por otro estudiodel mismo grupo RETIS encargado por el Ministerio de Justicia, esta vez dirigido a analizar la situación de la seguridad ciudadana en la franja fronteriza nacional (Ministerio da Justica e Cidadania/RETIS, 2016).

${ }^{5}$ Es, para poner un ejemplo, lo que Héctor Jaquet (2008) discutía sobre la situación generada en el binomio Posadas/Encarnación con la entrada del MERCOSUR: "En relación con el MERCOSUR, contrariamente a lo esperado, su implementación desde 1991-94 no trajo la integración de los pueblos tan declamada en los tratados, sino más bien vino a interrumpir y a añadir más nivel de conflicto a un marco histórico de relaciones entre los habitantes de las dos orillas. Las políticas integracionistas pronto marcaron la existencia de un MERCOSUR “desde arriba” y "para los de arriba” y otro que padecen "los de abajo". En términos de algunos agentes fronterizos, "un MERCOSUR de primera" que habría beneficiado a los grandes empresarios y a los circuitos transnacionales, y "un MERCOSUR de segunda”, que afectó notablemente a las poblaciones locales fronterizas periféricas” (p. 57).

6 "En el fondo llegamos aquí al hecho epistemológico fundamental de que categorías espaciales como el territorio, la residencia, la propiedad del suelo, pero simultáneamente el viaje, el nomadismo y el sedentarismo, son asimismo determinaciones constitutivas de la ciudadanía” (Balibar, 2013, p. 114) 


\section{Bibliografía}

Aduanas de Chile (2017). Estadísticas de 2017.Recuperado de: http:// www.aduana.cl/aduana/site/artic/20180405/asocfile/ 20180405132953/estadisticas2017.pdf

Anderson L., da Costa, E. yBenedetti, A. (2017). A feira livre de corumbá/ ms na fronteira Brasil-Bolívia.Bol. Geogr., Maringá, 35 (3), 93-108. DOI: 10.4025/bolgeogr.v35i3.28099

Agnew, J. y V. Oslander (2010). Territorialidades superpuestas, soberaníasen disputa: lección empírica desde América Latina. Tábula Rasa(13),191-213.Recuperado de: http://www.scielo.org.co/pdf/tara/ n13/n13a08.pdf

Augé, M. (1992). Los no lugares: Espacios del anonimato. Barcelona, España: Gedisa

Balibar, E. (2013). Ciudadanía, Buenos Aires, Argentina: Adriana Hidalgo Editora.

Barajas, M., Wong, P. y Oddone, N. (2015).Fronteras y procesos de integración regional. Ciudad de México, México: Juan Pablos Editor/el Colegio de la Frontera Norte.

Borja, J. y Castells, M. (1997). Local y global. Madrid, España: Taurus.

Brenner, N. yTheodore, N. (2002). Cities and the Geographies of Actually Existing Neoliberalism.Antipode, 34 (3). DOI: 10.1111/14678330.00246

Castells, M.(1989). La ciudad informacional. Madrid, España: Alianza Editorial.

Certeau, M. (2008). Andar la ciudad. Bifurcaciones:Revista de Estudios Culturales Urbanos, (7). Recuperado de: http://www.bifurcaciones.cl/ 007/colerese/bifurcaciones_007_reserva.pdf

Dahrendorf, R. (1979). Las clases sociales y su conflicto en la sociedad industrial. Madrid, España: EdicionesRialp.

Dear, M. y Leclerc, G. (2003). Postborder City: Cultural Spaces of Bajalta California. Londres, UK: University of Southern California.

Dilla, H. (2010). La frontera dominico-haitiana, Santo Domingo, República Dominicana: Ediciones Manatí.

Dilla, H. (2016). La regionalización precaria en la frontera dominico/haitiana. Si Somos Americanos. Revista de Estudios Transfronterizos, 16(1), 15-41. 
Dilla, H. y Álvarez, C. (2018). Economía e intercambio desigual en una región transfronteriza: Arica, Chile-Tacna, Perú. EstudiosFronterizos, 19 (9). DOI:10.21670/ref.1809009

Domínguez, A. (2010). La organización de las fronteras: etnografía de la cotidianidad fronteriza Chui/Chuy.En C. Casas y M. Ortolán (eds.) Miradas comparativas sobre las Fronteras en América Latina.Ciudad de México, México: Miguel Angel Porrúa.

Ferrari, M. (2013). Zona de fronteira, cidades gêmeas e interações transfronteiricas no contexto do MERCOSUL.Revista Transporte y Territorio(9), 87-104. Recuperado de: http:// revistascientificas.filo.uba.ar/index.php/rtt/article/view/305

Foucault, M. (2003). Vigilar y castigar.Ciudad de México, México: Siglo XXI Editores.

Foucher, M. (1991).Fronts etFrontieres. Paris, Francia: Fayard.

Fraser, N. (2003). La justicia social en la era de la política de la identidad.En Fraser N. y Honneth A.(eds.) ¿Redistribución o reconocimiento? Madrid, España: Ediciones Morata.

Glassner, M. y Blij, M. (1980).Systematic Political Geography, New York, EE.UU: John Wiley.

Harvey, D. (1996). Justice, Nature and the Geography of Difference.Massachusetts-Oxford, UK: Blackwell Publishers.

Harvey, D. (2014). Espacios del capital, Madrid, España:Akal.

Herzog, L. (1990). Where North Meets South. Austin, EE.UU: University of Texas Press.

Herzog,L. y Sohn, C. (2014). The Cross-Border Metropolis ina Global Age: A Conceptual Model and Empirical Evidence from the USMexico and European Border Regions.Global Society, 28:4, 441-461, DOI: $10.1080 / 13600826.2014 .948539$

Hurtado, A. y Aponte, J. (2017). ¿Hacia un gobierno transfronterizo?: explorando la institucionalidad para la integración colomboperuana.Estudios Fronterizos, 18 (35), 70-89.

Jaquet, H. (2008). Más allá de la frontera, las fronteras:Una aproximación socioespacial a las situaciones fronterizas de Misiones, Argentina.En H. Dilla (ed.), Ciudades en la Frontera, (pp. 31-66). Santo Domingo, República Dominicana: Editorial Manatí. 
Kearney, M., (2003). Fronteras y límites del Estado y el Yo al final del imperio. Alteridades, 13 (25), 47-62. Distrito Federal, México: Universidad Autónoma Metropolitana Unidad Iztapalapa. Recuperado de: https://www.redalyc.org/pdf/747/74702506.pdf

Kearney, M. (2008). La doble misión de las fronteras como clasificadoras y como filtros de valor. En L.Velazco, (ed.)Migración, fronteras e identidades étnicas transnacionales(pp. 79-116).Ciudad de México, México: Miguel Angel Porrúa.

Liberona, N. (2015). La frontera cedazo y el desierto como aliado. Prácticas institucionales racistas en el ingreso a Chile.Polis,Revista Latinoamericana, 14 (42), 143-165. Recuperado de: http://www.redalyc.org/ articulo.oa?id=30544552008

Luhman, N. (1990). Sociedad y sistema: la ambición de la teoría. Madrid, España: Paidós.

Machado de Olivera, T. (2005). Tipologia das relacoes frontereicas: elementos para o debate teorico-pratico. EmT.Machado de Olivera,(org.).Territorio sem limites(pp. 377- 408). Campo Grande, Brasil: Editorial UFMS.

Masi, F. yFalabella, G. (2005). Desarrollo económico regional em la frontera Paraguai-Brasil: estudio exploratorio del Alto Paraná. Em T.Machado de Olivera,(org.),Territorio sem limites(pp. 307-347). Campo Grande,Brasil: Editorial UFMS.

Mendoza, J. (ed.). (2007). El TLCAN y la frontera México-Estados Unidos. Foro internacional (181), 517-544. Ciudad de México, México: Miguel Angel Porrúa.Recuperado de: http://www.redalyc.org/ articulo.oa?id=59921013007

Meza, N. (2012). Espacios Regionales Fronterizos: Escenarios de Integración. Revista Integración \& Comercio, 34 (16), 25-32.

Mezzadra,S. y Neilson, B. (2013). Border as a Method or the Multiplication of Labor. Durham, EE-UU: Duke University Press.

Ministerio deIntegración Nacional y RETIS.(2005). Proposta do Programa de Desenvolvimiento da Faixa de Fronteira. Brasilia, Brasil:

Ministerio deJusticiay Cidadania y RETIS (2016). Segurança pública nas fronteiras, Brasilia, Brasil.

Morin, E. (2000).Sociología. Madrid, España:Tecnos.

Noriega, R. (2008). Tabatinga: una ciudad en la frontera de la Amazonía.En H. Dilla (ed.), Ciudades en la Frontera(pp. 97-126). Santo Domingo, 
República Dominicana: Editorial Manatí.

Nweihed, K. (1990). Frontera y límite en su marco mundial.Caracas, Venezuela:Equinoccio.

Oddone, N. y Galantini, E. (2015). Por la triple frontera más austral de América. EnM., Barajas, P. Wong y N. Oddone (eds.), Fronteras y procesos de integración regional. Ciudad de México, México: Juan Pablos Editor/el Colegio de la Frontera Norte.

Oddone, N., Quiroga, M., Sartori, H.yWilliner, A. (2016). Pactos Territoriales en la construcción de regiones transfronterizas. Santiago, Chile:Cepal.

Ohmae, K. (1991). El mundo sin fronteras. Madrid, España: McGraw-Hill/ interamericana de España.

Peña, S. (2008). Ciudades y Fronteras: los retos de la planificación transfronteriza. En H. Dilla (ed.), Ciudades en la Frontera(pp. 263283). Santo Domingo, República Dominicana: Editora Manatí.

Perkman, M. (2003). Cross-Border Regions in Europe: Significance and Drivers Of Regional Cross-Border Co-Operation. En European Urban and Regional Studies 10(2),153-171.Recuperadode: https:// dspace.lboro.ac.uk/dspace-jspui/bitstream/2134/660/4/ Perkmann_cross-border_regions_2003\%5B1\%5D.pdf

Prescott, J. R. V. (1987). Political Frontiers and Boundaries, New Yok, EE.UU: Routledge.

Ríos, A. (2017). The Border: a Double Sonnet. Recuperado de: https:// www.sopitas.com/801607-u2-alberto-rios-poema-concierto/

Rosière, S. y Jones, R. (2012).Teichopolitics: Re-considering Globalisation Through the Role of Walls and Fences.Geopolitics, 17 (1), 217-234. DOI: $10.1080 / 14650045.2011 .574653$

Ruiz, C. y Martínez G. (2015). Comercio informal transfronterizo MéxicoGuatemala desde una perspectiva de frontera permisiva. Estudios Fronterizos,nueva época, 16 (31), 149-174. Recuperado de: https:// www.redalyc.org/articulo.oa

Sassen, S. (2010).Territorios, autoridad y derechos: de los ensamblajes medievales a los ensamblajes globales. Buenos Aires, Argentina: Katz.

Sartori, G. (1999). Comparación y método comparativo. En G. Sartorio y L. Morlino(eds.), La comparación en las ciencias sociales(pp. 28-49). Madrid, España: Alianza Editorial. 
Silva, L. (2013). Redes de transporte de mercadorias e fronteira: a logísticanascidadesgêmeas de Corumbá (Brasil) e Porto Suárez (Bolívia). Revistatransporte y territorio, 9, 141-160.Recuperado de: https://www.redalyc.org/articulo.oa

Sohn, C. (2014). Modelling Cross-Border Integration: The Role of Borders as a Resource. Geopolitics, 19 (3),587-608. DOI: 10.1080/ 14650045.2014.913029

Tarrius, A. (2000). Leer, describir, interpretar las circulaciones migratorias. Relaciones,Estudios de Historia y Sociedad,21 (83). Zamora, México: El Colegio de Michoacán, A.C. Recuperado de:http:// www.redalyc.org/articulo.oa?id=13708303

Urdaneta, A.(2002). Vecindad en la frontera colombo/venezolana: situaciones de cooperación y conflicto y su incidencia en las relaciones bilaterales. En R. Silié y C. Segura (eds.), Hacia una nueva visión de las fronteras(pp. 105-126.). Santo Domingo, República Dominicana: Búho editorial.

Valero, M. (2004). Ciudad y fronteras. Aldea Mundo,9 (17), 120-144. Recuperado de: http://erevistas.saber.ula.ve/index.php/aldeamundo/ article/view/8818/0

Valero, M. (2008). Ciudades transfronterizas e interdependencia comercial en la frontera Venezuela/Colombia. En H. Dilla (ed.), Ciudades en la Frontera(pp. 69-95). Santo Domingo,República Dominicana: Editorial Manatí.

Valero, M. (2009). Redes urbanas transfronterizas: dos escenarios venezolanos.Aldea Mundo, Revista sobre Fronteras e Integración, 14 (27), 7-15. Recuperado de: http://redalyc.org/articulo.oa? id $=54315984002$

Zárate, C., Aponte, J. yVictorino, N. (2017). Perfil de una región transfronteriza en la Amazonia. Leticia, Colombia: Universidad nacional de Colombia. 